\title{
Indicadores para evaluar la rendición de cuentas en los medios de comunicación
}

\section{Indicators for evaluating media accountability \\ Indicadores para avaliar a prestação de contas na mídia}

Marcel Mauri-Rios, Universitat Pompeu Fabra, Barcelona, España (marcel.mauri@upf.edu) Xavier Ramon-Vegas, Universitat Pompeu Fabra, Barcelona, España (xavier.ramon@upf.edu) Ruth Rodríguez-Martínez, Universitat Pompeu Fabra, Barcelona, España (ruth.rodriguez@upf.edu)

Jesús Díaz-Campo, Universidad Internacional de La Rioja, Logroño, España (j.diaz@ unir.net)

RESUMEN | Esta investigación tiene como objetivo determinar indicadores clave para evaluar la capacidad de transparencia, autorregulación y participación de los usuarios, las tres dimensiones de la accountability. Se adoptó un triple punto de partida metodológico: revisión sistemática de literatura sobre indicadores de accountability y sus características, un mapeo de medios de referencia con indicadores de accountability, y evaluación de medios. Se obtuvo un sistema de 11 indicadores clave para medir y establecer comparaciones entre sitios web de medios de comunicación y determinar si satisfacen las tres dimensiones clave de la rendición de cuentas y los elementos asociados. Dicho sistema puede ser aplicado en múltiples escenarios para fomentar un periodismo de calidad.

PALABRAS CLAVE: rendición de cuentas; periodismo; ética; indicadores; transparencia; autorregulación; participación; usuarios; audiencia. 
ABSTRACT / This research aims to determine key indicators to assess the capacity for transparency, self-regulation, and user participation, accountability's three dimensions. We adopted a triple methodological starting point: a systematic review of literature on accountability indicators and their characteristics, a mapping of mainstream media that have accountability indicators, and media accountability assessment. We obtained a system of 11 key indicators to measure and compare media websites and determine whether they comply with the three key dimensions of accountability. This system can be applied in multiple settings to promote quality journalism.

KEYWORDS: accountability; journalism; ethics; indicators; transparency; self-regulation; participation; users; audience.

RESUMO|Esta pesquisa visa determinar indicadores-chave para avaliar a capacidade de transparência, autorregulação e participação dos usuários, as três dimensões da "prestação de contas". Um ponto de partida metodológico triplo foi adotado: uma revisão sistemática da literatura sobre indicadores de prestação de contas e suas características, um mapeamento dos meios de referência com indicadores de prestação de contas, e avaliação da mídia. Um sistema de 11 indicadores-chave foi obtido para medir e comparar sites de mídia e determinar se eles satisfazem as três dimensões-chaves da prestação de contas e os elementos associados. Tal sistema pode ser aplicado em múltiplos ambientes para fomentar o jornalismo de qualidade.

PALAVRAS-CHAVE: prestação de contas; jornalismo; ética; indicadores; transparência; autoregulação; participação, usuários; público. 


\section{INTRODUCCIÓN}

El periodismo experimenta múltiples transformaciones que inciden de forma directa sobre todas las fases del proceso informativo, incluyendo la producción, la difusión y el consumo de noticias. La revolución digital (Carlson \& Lewis, 2019) ha multiplicado las plataformas de difusión, las formas de presentar los contenidos, las posibilidades para obtener información, así como para ahondar en la relación con las audiencias. En un escenario complejo y en constante evolución, el periodismo enfrenta cambios estructurales de primera magnitud, como las turbulencias causadas por la crisis económica, el descenso de la inversión publicitaria y el colapso de los modelos de negocio tradicionales (Pérez-Soler \& Micó-Sanz, 2020; Waisbord, 2019). Asimismo, los ciclos informativos se han acelerado dramáticamente (Usher, 2018) y las empresas periodísticas se orientan hacia las métricas (Nelson, 2019). Los medios están "orientando progresivamente sus estrategias de producción y circulación hacia la recomendación, los rankings y otros tipos de algoritmos" (Nieborg \& Poell, 2018, p. 4280). Esto ha conducido, en muchas ocasiones, al aumento del clickbait y a la espectacularización (Ferrucci, 2020). Se suma la progresiva expansión de la desinformación (Amazeen, 2020), fenómeno que agudiza la crisis de confianza y de credibilidad del periodismo (Serazio, 2019).

En este contexto convulso, los profesionales de la información deben mantener la ética y la excelencia informativa como objetivos fundamentales (Christians et al., 2009). Según la teoría normativa de los medios, las empresas comunicativas son "actores morales, esto es, entidades dotadas de una consciencia e intencionalidad ética" (Luengo et al., 2017, p. 1147). Por ello, parte ineludible de su responsabilidad es involucrarse activamente en la tarea de fomentar la rendición de cuentas (accountability).

Este concepto hace referencia al "imperativo que obliga a un actor a responder, explicar y/o justificar su comportamiento ante otros individuos o instituciones" (Ramon-Vegas et al., 2020, p. 222). En el ámbito periodístico, esta acción enfatiza el "compromiso de los medios de responder por su práctica profesional ante la sociedad" (Rojas-Torrijos \& Ramon-Vegas, 2017, p. 916). La rendición de cuentas persigue "proteger y promover la libertad de expresión", "prevenir o limitar el daño que los medios puedan causar" y "promover beneficios positivos para la sociedad" (McQuail, 1997, p. 525). Está estrechamente vinculada con la responsabilidad social del periodismo y los periodistas para con la sociedad (Harcup, 2021; Lee \& Riffe, 2017). Como enfatiza Plaisance (2000), "rendir cuentas es de hecho ser responsable" (p. 260). Ramon-Vegas y Mauri-Ríos (2020) han concretado el concepto de accountability en los medios en tres dimensiones clave: transparencia, participación de la audiencia y autorregulación. 
Dimensión 1. Transparencia. Se refiere a la difusión pública de información corporativa (principios y valores, composición y estructura organizativa, situación financiera) y a explicar los estándares éticos, así como los procesos y decisiones editoriales de una forma clara y accesible (Craft \& Vos, 2021; Diakopoulos \& Koliska, 2017). En los últimos años, la preocupación por garantizar la transparencia informativa frente a la ciudadanía ha adquirido un rol central en múltiples ámbitos (Díez-Garrido et al., 2019).

Dimensión 2. Participación de la audiencia. Progresivamente, los medios abandonan la noción de la audiencia como un agente pasivo para fomentar su participación discursiva y creativa (López-Cepeda et al., 2019). La audiencia crea contenidos (produser) y participa a través de las plataformas digitales (Bruns, 2015; Pérez-Soler \& Micó-Sanz, 2020). En la era de la deliberación digital (Masip et al., 2019), se fomenta la relación directa con las audiencias en su creación, revisión y crítica de la información (Eberwein et al., 2011; Fuente-Cobos et al., 2014; Pérez-Díaz et al., 2020). Las audiencias pueden jugar un rol transformativo, corresponsabilizándose de la actividad de la información periodística de rendir cuentas (Culver, 2017). La voluntad de fomentar la participación es intrínseca al rol de los medios de comunicación, dada su "enorme responsabilidad no solo de informar de forma veraz, sino también de escuchar y dar voz a la ciudadanía" (Moreno-Gil, 2019, p. 55).

Dimensión 3. Autorregulación. Pone el énfasis en la capacidad de los medios y sus profesionales para establecer sus propias pautas de actuación y evitar la excesiva regulación por parte de los gobiernos (Marqués-Pascual \& GonzálezPeláez, 2020). Se concreta en códigos de conducta y otros mecanismos creados por los medios para garantizar una tarea periodística responsable (Fengler et al., 2014; Eberwein \& Porlezza, 2016).

Estas tres dimensiones se canalizan a través de un amplio abanico de instrumentos de rendición de cuentas. Para Bertrand (2018), son mecanismos impulsados por las empresas, periodistas, organizaciones y ciudadanos, sin intervención estatal (Eberwein et al., 2011) para velar por la responsabilidad de los medios y la calidad del producto informativo.

Este artículo busca ofrecer un sistema de indicadores que permita a los medios y a la ciudadanía en general (como receptor último de la función esencial del periodismo) determinar si el periodismo rinde cuentas de forma adecuada, con base en las tres dimensiones señaladas. 


\section{ESTADO DE LA INVESTIGACIÓN SOBRE INSTRUMENTOS DE RENDICIÓN DE CUENTAS EN LOS MEDIOS}

Gran parte de los estudios a nivel nacional e internacional examinan los códigos éticos y libros de estilo (Alsius, 1999; Aznar, 1999; Díaz-Campo \& ChaparroDomínguez, 2020; Marqués-Pascual \& González-Peláez, 2020; Wilkins \& Brenner, 2004), reconocidos como los mecanismos tradicionales con mayor relevancia para la práctica profesional, tal como lo muestran los resultados del proyecto Media Accountability and Transparency in Europe -MediaAcT- (Fengler et al., 2014). Esta investigación, coordinada por el Erich Brost Institute for International Journalism (EBI), encuestó a 1762 periodistas de 14 países europeos y del mundoárabe (Alemania, Austria, España, Estonia, Finlandia, Francia, Holanda, Italia, Jordania, Polonia, Reino Unido, Rumanía, Suiza y Túnez). Los libros de estilo fueron los instrumentos de accountability más bien valorados por los periodistas (3,74 sobre 5 puntos).

El estudio de los defensores del lector (ombudsman) también ha recibido una atención preferente en Europa y en los Estados Unidos (Ferrucci, 2019; Maciá, 2006; Nolan \& Marjoribanks, 2011; Ramon-Vegas et al., 2019, van Dalen \& Deuze, 2006). Las funciones de otros instrumentos tradicionales, como la crítica de medios (Fengler, 2003), las cartas al director (Pastor, 2010; Raeymaeckers, 2005; WahlJorgensen, 2002) o los consejos de la información (Mauri-Ríos et al., 2018; SuárezVillegas, 2015) también han sido extensamente abordadas.

Como señala Plaisance (2000), rendir cuentas es un fenómeno en constante evolución. Trabajos recientes estudian las nuevas posibilidades en el entorno en línea, como los blogs de crítica mediática (Cheruiyot, 2017), la crítica en las redes sociales (Eberwein et al., 2011), los comentarios sobre el contenido publicado (Craft et al., 2016), los sistemas de notificación y corrección de errores (Moreno-Gil, 2019), los libros de estilo dinámicos en Twitter (Rojas-Torrijos \& Ramon-Vegas, 2017) y los mecanismos de transparencia corporativa (Campos-Domínguez \& RedondoGarcía, 2015). Estos mecanismos innovadores inyectan a la profesión nuevas vías para incentivar la transparencia, la autorregulación y la participación, ayudando a superar las limitaciones asociadas con los instrumentos tradicionales.

Otras investigaciones mapean los instrumentos existentes en el contexto español. Alsius etal. (2011) ofrecen una panorámica de los mecanismos tradicionales, complementada por radiografías subsiguientes sobre los instrumentos innovadores (García-Avilés, 2019; Mauri-Ríos \& Ramon-Vegas, 2015). Rodríguez-Martínez et al. (2017) analizaron 60 mecanismos creados de forma exógena a los medios en las comunidades autónomas de Cataluña, Galicia, Madrid y Valencia. La historia y peculiaridades de las iniciativas de la rendición de cuentas en el contexto catalán fueron abordadas por Almiron y sus colegas (2016). Asimismo, se ha impulsado 
el mapeo de los instrumentos de accountability en áreas especializadas, como el periodismo deportivo (Ramon-Vegas \& Rojas-Torrijos, 2017). Ramon-Vegas y MauriRíos (2020) completaron el mapa de instrumentos de accountability en el panorama español, focalizándose en analizar la percepción de la ciudadanía y cómo rendir cuentas facilita la participación.

\section{OBJETIVOS Y METODOLOGÍA}

Pese a la extensa literatura existente sobre la rendición de cuentas en medios de comunicación, no se han desarrollado investigaciones que especifiquen qué indicadores pueden servir para evaluar las tres dimensiones de la accountability: transparencia, participación de la audiencia y autorregulación. Este trabajo busca subsanar este vacío, presentando un sistema de indicadores estandarizado que permita identificar en qué medida un medio es accountable. Dicho sistema también permite cuantificar estos parámetros y comparar sitios web de diferentes medios para determinar cuáles los satisfacen de forma más adecuada. Como objetivo complementario, el estudio recopila los casos más relevantes de medios a nivel internacional que incluyen indicadores de accountability, con especial hincapié en mapear los instrumentos existentes en el sistema mediático español.

Para desarrollar estos indicadores, se adoptó una estrategia metodológica en tres etapas. Primero, se realizó una revisión sistemática de literatura sobre rendición de cuentas, identificando los indicadores de accountability internos a los medios de comunicación existentes en el panorama internacional y determinar sus características. A partir de las referencias académicas clave detectadas (Bastian, 2019; Bertrand, 2018; Domingo \& Heikkilä, 2012; Eberwein et al., 2018; Fengler, 2003; Fengler et al., 2014; Fengler et al., 2015; García-Avilés, 2019; Hallin \& Papathanassopoulos, 2002; Pérez-Diaz et al., 2020; Silva \& Paulino, 2007), se formalizó un sistema de dimensiones e indicadores para evaluar la rendición de cuentas de un medio (tabla 1). Para sistematizar estos indicadores, se consideró y completó el mapa de instrumentos de rendición de cuentas en el escenario español elaborado por Ramon-Vegas y Mauri-Ríos (2020). Nuestra investigación define los instrumentos de rendición de cuentas detectados en la literatura y les asigna un sistema de puntuación que permite evaluar el grado de accountability de un medio.

En segundo lugar, se realizó un mapeo de medios que cuentan con alguno de los indicadores mencionados en las tres áreas geográficas de referencia de desarrollo de iniciativas de accountability: Europa, América del Norte y América Latina (Bastian, 2019; Eberwein et al., 2018). Se seleccionaron medios de referencia de esos territorios donde tradicionalmente estos instrumentos de rendición de cuentas han contado con un mayor arraigo y desarrollo (Ramon-Vegas et al., 2016). 
Dimensión 1. Transparencia

\begin{tabular}{cc}
\hline 1.1 & Blog de la redacción. \\
\hline 1.2 & Apartado web con información corporativa. \\
\hline 1.3 & $\begin{array}{c}\text { Otras herramientas de transparencia: listas abiertas de temas en producción (open } \\
\text { news lists), videos explicativos de decisiones editoriales, retransmisión en línea de los } \\
\text { consejos de redacción. }\end{array}$ \\
\hline 2.1 & Dimensión 2. Autorregulación \\
\hline 2.2 & Defensor del lector/audiencia. \\
\hline 2.3 & $\begin{array}{c}\text { Otras herramientas de autorregulación: sección, espacio o blog elaborado por } \\
\text { periodistas dedicado a la reflexión crítica de los contenidos publicados por otros } \\
\text { medios. }\end{array}$ \\
\hline 3.1 & Comentarios de los usuarios en las noticias publicadas en la web del medio. \\
\hline 3.2 & Comentarios de los usuarios en las redes sociales. \\
\hline 3.3 & Botones de corrección de errores. \\
\hline 3.4 & Contribuciones de los usuarios en la creación y revisión de los contenidos. \\
\hline & Corramientas de participación: cartas al director, chats y encuentros digitales con \\
\hline & los lectores, buzón para el envío de material confidencial. \\
\hline
\end{tabular}

Tabla 1. Dimensiones e indicadores para evaluar la rendición de cuentas de un medio

Fuente: Elaboración propia.

Estas se localizaron a través de un doble sistema. Por una parte, se empleó un muestreo no probabilístico por conveniencia (Ruiz Olabuénaga et al., 1998) entre medios de referencia de distintos países de las tres áreas referenciadas en la literatura revisada. Por otra parte, se empleó la estrategia de muestreo de bola de nieve (Goodman, 1961) a partir de sujetos o experiencias que aparecen en la literatura revisada. Partiendo de una pequeña cantidad de medios, se localizan otros casos. La selección también buscó ofrecer medios de todas las tipologías: periódicos en papel, medios digitales nativos, que desde su génesis incorporaron mecanismos de accountability (Benson, 2018), radios y televisiones (tabla 2). Ningún medio presenta todos los indicadores de rendición de cuentas aquí incluidos. Sin embargo, el mapeo de medios permitió identificar modelos de referencia para cada uno de los indicadores. 


\begin{tabular}{|c|c|c|}
\hline Territorio & País & Medio \\
\hline \multirow{8}{*}{ América del Norte } & \multirow{7}{*}{ Estados Unidos } & The New York Times \\
\hline & & The Washington Post \\
\hline & & Associated Press \\
\hline & & National Public Radio - NPR \\
\hline & & ProPublica \\
\hline & & MinnPost \\
\hline & & Texas Tribune \\
\hline & Canadá & The Globe and Mail \\
\hline \multirow{5}{*}{ América Latina } & Brasil & O Globo \\
\hline & Ecuador & El Comercio \\
\hline & Chile & El Mercurio \\
\hline & Colombia & El Tiempo \\
\hline & Uruguay & El País \\
\hline \multirow{15}{*}{ Europa } & \multirow{3}{*}{ Reino Unido } & The Guardian \\
\hline & & BBC \\
\hline & & The Economist \\
\hline & Italia & La Reppublica \\
\hline & Bélgica & De Staandard \\
\hline & \multirow{10}{*}{ España } & El País \\
\hline & & El Mundo \\
\hline & & La Vanguardia \\
\hline & & $A B C$ \\
\hline & & El Periódico de Catalunya \\
\hline & & ARA \\
\hline & & ElDiario.es \\
\hline & & La Marea \\
\hline & & Fíltrala \\
\hline & & RTVE \\
\hline
\end{tabular}

Tabla 2. Áreas geográficas y medios de referencia con algún indicador de accountability

Fuente: Elaboración propia. 
Puntuación

\begin{tabular}{cc}
\hline $0-5$ & Escasa orientación a rendir cuentas. \\
\hline $6-10$ & Favorece parcialmente rendir cuentas. \\
\hline $11-15$ & Fomenta activamente rendir cuentas. \\
\hline
\end{tabular}

Tabla 3. Escala de evaluación de rendición de cuentas de los medios

Fuente: Elaboración propia.

Tras elaborar el listado de indicadores de rendición de cuentas y detectar los medios de referencia que los disponen, se aplicó la metodología de obtención y documentación de indicadores (Codina, 2000) para articular un sistema que permita medir el grado de accountability de un medio de comunicación. Para cada indicador se establecieron categorías de análisis:

- Presentación y características

> Definición

> Características específicas

> Modelos de referencia

- Evaluación

> Examen

> Puntuación

> Observaciones

Se presenta la propuesta de puntuación para evaluar los medios analizados en la tabla 3.

El sistema propuesto garantiza: (1) la operatividad de los indicadores, es decir, que los elementos a analizar sean efectivamente medibles o evaluables; (2) la transparencia y la intersubjetividad de cada indicador, y (3) la replicabilidad o extensión de tales indicadores por parte de otros equipos de investigación.

\section{RESULTADOS}

\section{Dimensión 1. Transparencia}

Indicador 1.1. Blog de la redacción

- Definición: el medio cuenta con un blog o espacio destinado a profundizar en los procesos de producción informativa. Se puede utilizar este espacio para presentar las novedades del proyecto editorial. Así, se fomenta la transparencia de la tarea periodística y se abre un proceso de reflexión pública sobre esta. 
- Características específicas: para optimizar su buen funcionamiento como instrumento de rendición de cuentas, se debe observar:

> Difusión de la existencia del instrumento.

> Regularidad y actualización constante de publicación.

> Explicación de las novedades del proyecto editorial y comentario sobre coberturas desarrolladas.

> Necesidad de delimitar con claridad las entradas dedicadas a los procesos informativos y aquellas centradas en la promoción cruzada de los productos del medio.

> Posibilidad para los lectores de comentar y respuesta de la redacción.

- Modelos de referencia: algunos blogs clave son los impulsados por The Guardian (https://www.theguardian.com/help/insideguardian), Eldiario. es (http://www.eldiario.es/redaccion/) y El País, con su bitácora El País que hacemos (https://elpais.com/agr/el_pais_que_hacemos/a/).

\section{Evaluación}

- Examen: ¿Existe en el medio un blog de la redacción u otro espacio de transparencia editorial?

- Puntuación: 0/0,5/1 (0: no existe este espacio; 0,5: el espacio existe, pero no cumple varias de sus características esenciales; 1 : el espacio existe y cumple las características explicitadas).

\section{Indicador 1.2. Apartado en la web con información corporativa}

- Definición: en su sitio web, el medio debe aportar información corporativa que permita a los usuarios conocer de forma clara los principios editoriales y las características empresariales bajo las cuales desarrolla su actividad informativa.

- Características específicas: el apartado debe contenerla siguienteinformación:

1. Audiencia: información sobre las cifras de audiencia.

2. Año de fundación y trayectoria: año de fundación e historia del medio, coberturas relevantes y cambios de propiedad.

3. Titularidad y pertenencia a un grupo mediático: información sobre la titularidad pública o privada. Especificar la pertenencia a un grupo de comunicación y la relación con otras empresas. 
4. Cuentas públicas y estados financieros: información fácilmente accesible sobre las cuentas de resultados anuales y estados financieros del medio. También se puede detallar el modelo de financiación y las fuentes de ingresos del modelo de negocio. Si el medio recibe un subsidio o ayuda estatal, especificar la cantidad y los motivos que lo justifican.

5. Misión, objetivos y tendencia editorial: información sobre la tendencia ideológica y el posicionamiento editorial del medio, así como su misión.

6. Organización/miembrosdela redacción y dela dirección:información sobre la estructura del medio (departamentos, secciones), sobre los profesionales (biografía, imagen, correo electrónico o enlace a sus publicaciones e información sobre sus perfiles en redes sociales) y los miembros del consejo directivo.

Para rendir cuentas de forma eficaz, los medios deben incluir esta información en su sitio web. La inclusión de estos datos en la web de un grupo de comunicación dificulta su localización por parte de los usuarios.

- Modelos de referencia: las secciones About Us de MinnPost (www.minnpost.com/about/), ProPublica (www.propublica.org/about/) y The Texas Tribune (www.texastribune.org/about/) proporcionan un amplio abanico de elementos de transparencia corporativa. El periódico digital Eldiario.es (https://www.eldiario.es/escolar/periodismo-servicio-publicocuentas-eldiarioes_6_907169284.html) detalla de forma clara sus cuentas. A través de la sección Memória (http://memoria.oglobo.globo.com), el medio brasileño 0 Globo presenta una panorámica de su historia a través de los años.

\section{Evaluación}

- Examen: ¿Dispone el medio de una sección en su sitio web en la que se aporte información corporativa?

- Puntuación: 0-3 (0,5 puntos por cada uno de los ítems descritos).

\section{Indicador 1.3. Otras herramientas que fomentan la transparencia}

\section{Presentación y características}

- Definición: existencia de otras herramientas innovadoras implementadas por el medio, como las listas abiertas de temas en producción (open news lists), videos que expliquen las decisiones editoriales, retransmisión en línea de los consejos de redacción u otros espacios para explicar los procesos editoriales. 
- Características específicas: para optimizar su buen funcionamiento como instrumento de rendición de cuentas, las herramientas de transparencia deben observar los siguientes aspectos:

> Difusión de la existencia del instrumento.

> Uso y actualización constantes.

> Posibilidad de consultar la información de forma posterior en un repositorio o archivo alojado en la web del medio.

- Modelos de referencia: hasta el momento, este tipo de herramientas han sido empleadas por medios como The Guardian (https://www.theguardian. com/news/series/open-newslist) o La Repubblica (http://video.repubblica. it/rubriche/repubblica-domani/).

\section{Evaluación}

- Examen: ¿El medio ha desarrollado otras herramientas de transparencia?

- Puntuación: 0/1 (0: no existen otras herramientas; 0,5: existen otras herramientas, pero no cumplen varias de sus características esenciales -por ejemplo: regularidad de uso y actualización-; 1: sí existen otras herramientas y cumplen las características explicitadas).

- Observaciones: en caso de existir, explicar la naturaleza y el funcionamiento de dichas herramientas de transparencia.

\section{Dimensión 2. Autorregulación}

\section{Indicador 2.1. Defensor del lector/audiencia}

- Definición: la figura del ombudsman (o defensor) se inscribe claramente entre los mecanismos de los que se dotan los medios de comunicación para ejercer su labor con un mejor nivel ético. Debe recoger las quejas de los lectores, oyentes o espectadores sobre diversos aspectos de fondo o de forma, hablar con los periodistas y productores, y dar una respuesta pública a través de un blog o sección específica.

- Características específicas: para optimizar su buen funcionamiento como instrumento de rendición de cuentas, se deben observar los siguientes aspectos:

> Difundir la existencia de esta figura.

> Regularidad constante de publicación y actualización de su espacio. 
> Indicar de forma clara el procedimiento para enviar quejas, comentarios y sugerencias.

> Debatir cuestiones vinculadas con la ética y la calidad periodística.

> Se recomienda que el plazo de ejercicio no sobrepase los tres años.

- Modelos de referencia: en general, los medios que han incorporado esta figura suelen contarse entre los más prestigiosos de sus respectivos países. Existe un listado de los miembros de la ONO (Organization of News Ombudsmen) y los medios que representan en https://www.newsombudsmen.org/regularmembers/. Son referentes en el terreno internacional el blog Open Door de The Guardian (https://www.theguardian.com/commentisfree/series/ open-door) y el blog de Elizabeth Jensen en NRP (https://www.npr.org/ sections/ombudsman/).

\section{Evaluación}

- Examen. ¿Cuenta el medio con un defensor del lector/audiencia?

- Puntuación: 0/0,5/1 (0: el medio no dispone de defensor; 0,5: el medio dispone de defensor, pero su espacio no está actualizado; 1: el medio dispone de un espacio actualizado de defensor del lector/audiencia).

\section{Indicador 2.2. Libro de estilo del medio de comunicación}

- Definición: documento que establece las recomendaciones lingüísticas (corrección sintáctica y léxica) o estilísticas (preferencias del medio en cuanto al modo de escribir), así como los procedimientos y recursos de comportamiento profesional y deontológicos a seguir en una empresa periodística para desarrollar un producto de calidad.

- Características específicas: para optimizar su buen funcionamiento como instrumento de rendición de cuentas, se deben observar los siguientes aspectos:

> Difusión de la existencia del instrumento.

> El libro de estilo debe atender a su doble función.

> El libro de estilo debe estar actualizado, adaptarse a cuestiones vinculadas con el entorno digital y las redes sociales.

> El librode estilo no puede ser un instrumento de circulación restringida y debe ser accesible a través del sitio web del medio. 
> Idealmente, ofrecer la posibilidad de que los usuarios participen del proceso de revisión y actualización.

> Como complemento, promover la implantación de libros de estilo dinámicos en redes sociales.

- Modelos de referencia: The New York Times con su Ethical Journalism. A Handbook of Values and Practices for the News and Editorial Departments (https://www.nytimes.com/editorial-standards/ethical-journalism.html); BBCEditorialGuidelines(http://www.bbc.co.uk/guidelines/editorialguidelines/), o el Manual de Estilo de RTVE (http://manualdeestilo.rtve.es) son referencias a considerar. El Manual de Buenas Prácticas de El Comercio (Ecuador) (http://www.grupoelcomercio.com/images/pdf/redes.pdf) proporciona guías específicas sobre la actuación periodística en las redes sociales. Algunos medios pioneros en la introducción de libros de estilo dinámicos en Twitter son The Guardian (@Guardianstyle), The Economist (@Econstyleguide) oAP (APStylebook).

\section{Evaluación para medios de comunicación}

- Examen: ¿Cuenta el medio con un código deontológico o libro de estilo?

- Puntuación: 0/1,5/3 (0: el medio no dispone del instrumento; 1,5: el medio dispone de libro de estilo, pero no es accesible en línea; 3: el medio dispone del instrumento y se encuentra accesible en línea).

\section{Indicador 2.3. Otros instrumentos de autorregulación}

- Definición: el medio dispone de otros instrumentos de autorregulación, como por ejemplo una sección, espacio o blog elaborado por periodistas dedicado a la reflexión crítica de los contenidos publicados por otros medios.

- Características específicas:

> Para optimizar su función de rendición de cuentas, esta sección o espacio no solo debe abordar novedades del sector, informaciones sobre audiencias o focalizarse en personalidades/celebrities, sino que debe también promover el debate y la reflexión crítica sobre los contenidos periodísticos publicados por otros medios de comunicación.

> Se debe asegurar la regularidad de publicación de los contenidos.

- Modelos de referencia: la sección de Media de The New York Times (https://www.nytimes.com/topic/subject/media) es un ejemplo relevante a nivel internacional. Pareu Màquines en Ara favorece la crítica sobre medios 
(https://www.ara.cat/etiquetes/pareu_maquines.html), complementando las columnas de Mònica Planas (https://www.ara.cat/firmes/monica_ planas/) sobre crítica televisiva y reflexión periodística.

\section{Evaluación}

- Examen: ¿Existen una sección, espacio o blog dedicado a la crítica de medios?

- Puntuación: 0/0,5/1 (0: no existe este espacio; 0,5: el espacio existe, pero no cumple varias de sus características esenciales; 1 : sí existe este espacio).

\section{Dimensión 3: Participación de los usuarios}

Indicador 3.1. Comentarios de los usuarios en las noticias publicadas en la web del medio

\section{Presentación y características}

- Definición: el medio permite a los usuarios comentar las informaciones publicadas, leer y responder a los comentarios efectuados previamente.

- Características específicas: para optimizar su buen funcionamiento como instrumento de rendición de cuentas, se deben observar los siguientes aspectos:

> Ofrecer la posibilidad a los usuarios de comentar las noticias a partir de un texto abierto. También se puede ofrecer que valoren las noticias según una escala de puntuación.

> Disponer de una política de publicación que guíe las intervenciones de los usuarios. Esta debe incluir directrices como prohibir utilizar lenguaje ofensivo, publicar publicidad o desviarse del tema objeto de debate. Indicar si el usuario debe estar registrado o ser subscriptor para participar.

> Implementar un sistema de moderación eficaz.

- Modelos de referencia: las normas de participación de medios como El País (https://elpais.com/estaticos/normas-de-participacion), Eldiario.es (https:// www.eldiario.es/participación/) y Elmercurio.com (https://static.emol.cl/ emol50/pdf/TyC-Comentarios-Emol.pdf), accesibles desde el espacio de comentarios de cada noticia, representan buenos ejemplos de normas que guían las contribuciones de los usuarios.

\section{Evaluación}

- Examen: ¿Puede el usuario comentar las informaciones publicadas? 
- Puntuación: 0/0,5/1 (0: no se puede realizar esta acción; 0,5: se puede realizar esta acción, pero los comentarios no están moderados o el usuario no debe estar registrado para participar; 1: se puede realizar esta acción y existe una moderación y la necesidad que el usuario esté registrado o sea subscriptor para participar).

\section{Indicador 3.2. Comentarios de los usuarios en las redes sociales}

\section{Presentación y características}

- Definición: el medio dispone de perfiles en las principales redes sociales (Twitter y Facebook) y permite a los usuarios emitir comentarios, sugerencias y quejas a través de estas. Además de los perfiles generales, puede disponer de canales específicos para permitir la participación de los usuarios.

- Características específicas: para optimizar su buen funcionamiento como instrumento de rendición de cuentas, se deben observar los siguientes aspectos:

> Difundir la existencia del instrumento.

> Utilizar las redes sociales no únicamente como canal de difusión o de promoción de contenidos. Los medios/periodistas deben dar respuesta a la crítica para que las redes sean un instrumento efectivo y real de rendir cuentas.

- Modelos de referencia: medios como The Washington Post (http://www. washingtonpost.com/wp-srv/guidelines/social-media.html) o The New York Times (https://www.nytimes.com/2017/10/13/reader-center/social-mediaguidelines.html) disponen de políticas específicas que contemplan a las redes sociales como espacio de participación, debate y reflexión periodística.

\section{Evaluación}

- Examen: ¿El medio permite a los usuarios participar en las redes sociales?

- Puntuación: 0/1 (0: el medio no utiliza las redes con finalidades de accountability; 1: el medio utiliza activamente las redes sociales con finalidades de accountability).

\section{Indicador 3.3. Botones de corrección de errores}

- Definición: el medio permite a los usuarios notificar los errores detectados en las piezas periodísticas mediante un botón, formulario o correo electrónico. Así, demuestra su capacidad de rendir cuentas por los errores cometidos. 
- Características específicas: para optimizar su buen funcionamiento como instrumento de rendición de cuentas, se deben observar los siguientes aspectos:

> Sistematicidad: asegurar la existencia de un botón de notificación en cada una de las piezas publicadas.

> Localización: asegurar que la posición (superior o inferior de la noticia) sea constante y fácilmente identificable por parte de los usuarios.

> Especificar los datos requeridos para la identificación de los usuarios.

> Posibilidad de detallar el tipo de error detectado (ético, datos o tipográfico, problema de moderación, derechos y permisos, otros).

> Facilitar una extensión suficiente para notificar el error.

> El medio puede sistematizar los errores y rectificaciones en un espacio alojado en su sitio web. Este debe mantenerse debidamente actualizado.

- Modelos de referencia: medios como De Staandard en Bélgica, El Tiempo en Colombia o El País en Uruguay disponen de esta herramienta. En Estados Unidos, The Texas Tribune (https://www.texastribune.org/corrections/) y ProPublica (https://www.propublica.org/corrections/) cuentan con una página que recoge los errores y correcciones efectuadas. También El Mercurio, en Chile, incorpora al final de cada noticia esta referencia: “Encontraste algún error? Avísanos".

\section{Evaluación}

- Examen: ¿Existe un sistema de detección y corrección de errores?

- Puntuación: 0/1 (0: el medio no dispone del mecanismo; 1: el medio dispone del mecanismo).

\section{Indicador 3.4. Contribuciones de los usuarios en la creación y revisión de los contenidos}

- Definición: el medio cuenta con un canal dedicado a los contenidos escritos, audiovisuales o multimedia generados por los usuarios (User Generated Content).

- Características específicas: para optimizar su buen funcionamiento como instrumento de rendición de cuentas, se deben observar los siguientes aspectos:

> Difundir la oportunidad de contribución de los usuarios en la creación de los contenidos periodísticos. 
> Indicar con claridad el procedimiento para enviar contribuciones.

> Sugerir aquellos temas, enfoques y fuentes prioritarias para el medio.

> Para optimizar el valor de este instrumento, se debe ir más allá de una simple recolección de imágenes u opiniones, como predomina en páginas como Community, en The Guardian (https://www.theguardian.com/community), que sustituye otra herramienta del mismo periódico que perseguía el mismo objetivo: GuardianWitness (https://www.theguardian.com/help/ insideguardian/2018/aug/21/guardianwitness-is-closing-but-youcan-still-contribute-your-stories ).

> Regularidad de publicación y actualización de la sección o canal.

> Posibilidad de comentarios de otros lectores y debate activo con los periodistas.

- Modelos de referencia: Se pueden destacar ejemplos como Eu Reporter de O Globo (https://oglobo.globo.com/eu-reporter/) o Los socios/as escriben de La Marea (https://www.lamarea.com/secciones/los-socios-escriben/). La sección TribTalk de The Texas Tribune (https://www.tribtalk.org) -ahora ya cerrada- fue referente en publicar artículos en profundidad sobre los ejes informativos tratados por el medio.

\section{Evaluación para medios de comunicación}

- Examen: ¿Existe una sección dedicada al contenido enviado por los usuarios?

- Puntuación: 0/1 (0: el medio no dispone de esta sección; 1: el medio dispone de esta sección).

\section{Indicador 3.5. Otras herramientas de participación}

- Definición: el medio ha implementado otras herramientas que dan la posibilidad a los usuarios de contactar con los redactores para expresar su percepción sobre el contenido publicado o para contribuir al desarrollo de nuevas coberturas periodísticas. Algunas tipologías relevantes de herramientas son:

> Cartas al director: existencia de una sección de cartas al director, donde los usuarios puedan mandar y ver respondidas sus sugerencias y quejas por el tratamiento informativo efectuado. Las cartas al director también son un espacio idóneo para discutir temas de interés general. 
Chats y encuentros digitales con los lectores: el medio permite a los usuarios contactar con el equipo editorial a través de chats y encuentros digitales (en formato escrito o video-chat) para conversar en tiempo real sobre la información publicada u otras cuestiones sobre el proceso periodístico.

> Buzones para enviar material confidencial: sistemas que permiten mandar de forma segura pistas o material confidencial al medio, con el objetivo de contribuir al desarrollo de una investigación periodística.

- Características específicas: difundir la existencia del instrumento. Indicar con claridad el procedimiento para enviar contribuciones, que debe ser sencillo.

- Modelos de referencia:

> Cartas al director: espacios frecuentes en medios , como por ejemplo The New York Times (https://www.nytimes.com/section/opinion/ letters), The Globe and Mail (https://www.theglobeandmail.com/ opinion/letters/)o El Mercurio, con la sección Blogs, cartas y opinión (https://www.elmercurio.com/blogs/).

> Chats y encuentros digitales con los lectores: The Washington Post, con sus Q\&A, es referencia en la celebración y archivo sistemático de encuentros digitales (http://live.washingtonpost.com/). El periódico chileno El Mercurio celebra encuentros virtuales, pero también presenciales, entre equipo editorial, expertos externos al medio y los lectores (https://encuentros.elmercuio.com).

> Buzones para enviar material confidencial: The New York Times permite a los usuarios mandar pistas mediante varios canales (WhatsApp, Signal, correo electrónico y postal, SecureDrop). En España, el sistema equivalente es Fíltrala (https://filtrala.org/), utilizado por medios como Eldiario.es o La Marea. Ara dispone de una herramienta propia con el mismo propósito (https://www.ara.cat/investigacio.html).

\section{Evaluación}

- Examen: ¿Dispone el medio de otras herramientas de participación?

- Puntuación: 0/0,5/1 (0: el medio no dispone de otras herramientas de participación; 1: el medio dispone de otras herramientas de participación).

- Observaciones: en caso de existir, se debería explicar la naturaleza y el funcionamiento de dicho espacio o herramienta. 


\section{DISCUSIÓN}

Este trabajo confirma la necesidad de profundizar en el estudio académico sobre la rendición de cuentas o media accountability, una de las cuestiones clave del periodismo actual (Bertrand, 2018; Eberwein et al., 2018). Entre los diversos aportes derivados de la investigación se observa, en primer lugar, la viabilidad de formalizar una propuesta de categorías de análisis que incluya las tres dimensiones que definen la rendición de cuentas: transparencia, autorregulación y participación de la audiencia.

Esta contribución parte de los mapeos previos de indicadores existentes que contribuyen a la rendición de cuentas de los medios (Bastian, 2019; Bertrand, 2018; Domingo \& Heikkilä, 2012; Eberwein et al., 2018; Fengler, 2003; Fengler et al., 2014; Fengler et al., 2015; García-Avilés, 2019; Hallin \& Papathanassopoulos, 2002; Pérez-Diaz et al., 2020; Ramon-Vegas \& Mauri-Ríos, 2020; Silva \& Paulino, 2007) y propone un modelo para medir el grado de accountability de un medio. La herramienta analítica permite conocer en qué medida la rendición de cuentas está presente o no en los medios de comunicación, y cuantificar si esta presencia es escasa o si está implementada de forma adecuada.

La propuesta incide en la necesidad de potenciar la conexión entre el ámbito académico y el ejercicio práctico de la profesión. La correlación advertida entre la literatura existente sobre rendición de cuentas y la presencia de los indicadores propuestos en medios de referencia confirma ese vínculo. Por otro lado, si bien cada sistema mediático cuenta con características propias, la presencia de indicadores comunes que garantizan las dimensiones de transparencia, la autorregulación y la participación de la audiencia en medios de Europa, América del Norte y América Latina (Bastian, 2019; Eberwein et al., 2018; Fengler et al., 2015; Silva \& Paulino, 2007) confirma la aceptación similar que existe del concepto de rendición de cuentas en contextos mediáticos occidentales. Este aporte, derivado de este análisis, plantea la necesidad de futuras investigaciones que incluyan áreas geográficas diferentes para conocer la idoneidad de su aplicación en otras culturas periodísticas.

\section{CONCLUSIONES Y ESCENARIOS DE APLICACIÓN}

La rendición de cuentas de la información periodística adquiere una importancia capital en el escenario actual. Los estudios que examinan las características y las funciones de los instrumentos tradicionales e innovadores, así como aquellos que mapean su existencia o ausencia, deben dar paso a estudios más complejos, que evalúen de forma pormenorizada la capacidad de los medios para responder a la transparencia, la participación de la audiencia y a la autorregulación, contribuyendo 
también a la toma de decisiones por parte de las empresas de comunicación. La metodología de análisis propuesta puede ser empleada para que académicos y profesionales analicen medios de comunicación, al permitir observar la cantidad y calidad de los indicadores presentes en un sitio web, identificar los más utilizados y monitorizar la aparición de nuevos mecanismos.

La investigación aporta a la literatura existente al entregar una propuesta plausible y útil para futuros estudios. Estos indicadores pueden ser de gran utilidad para análisis académicos de medios de comunicación, ya que pueden proporcionar resultados comparativos sobre su presencia en medios con características comunes o entre medios de diferentes regiones y culturas periodísticas. Esto puede ayudar a identificar tendencias, similitudes, diferencias, fortalezas y debilidades de los medios analizados.

En lo referido al ámbito profesional, esta propuesta puede ser empleada por directivos de medios de comunicación para determinar si el sitio web de su organización está adaptado de forma adecuada a los requerimientos de transparencia, participación de los usuarios y autorregulación o si necesita incorporar mejoras. Asimismo, esta metodología se puede tomar como modelo de referencia para crear o rediseñar sitios web de medios que deseen tener en cuenta el concepto de media accountability.

Por otra parte, esta metodología de análisis aplicada a los medios puede ser de utilidad en los siguientes escenarios:

- Escenario 1: permitir a los cargos de decisión de los medios de comunicación reflexionar y tomar decisiones sobre la introducción o rediseño de sus instrumentos de rendición de cuentas, en aras de mejorar su transparencia, autorregulación y participación de la audiencia.

- Escenario 2: fomentar los análisis académicos y profesionales de los medios de comunicación. Las plantillas permiten evaluar la presencia o inexistencia de instrumentos que facilitan la rendición de cuentas de los medios. En el marco de un territorio o región, se puede observar el grado de implantación y la naturaleza de los instrumentos presentes en una serie de medios e identificar los más utilizados. Esto puede ayudar a identificar tendencias, similitudes, diferencias, fortalezas y debilidades de los medios analizados.

- Escenario 3: estas plantillas posibilitan medir y establecer comparaciones entre medios de comunicación de diferentes culturas periodísticas para determinar en qué medida los contextos mediáticos influyen sobre la incorporación de instrumentos que satisfacen adecuadamente las tres dimensiones clave de la rendición de cuentas. 


\section{FINANCIAMIENTO}

Esta investigación forma parte del proyecto "Instrumentos de rendición de cuentas ante la desinformación: Impacto de las plataformas de fact-checking como herramientas de accountability y propuesta curricular (FACCTMedia)", financiado por el Ministerio de Ciencia e Innovación del Gobierno de España (PID2019- 106367GBIO0 /AEI/10.13039/501100011033).

\section{REFERENCIAS}

Almiron, N., Narberhaus, M., \& Mauri-Ríos, M. (2016). Mapping media accountability in stateless nations: the case of Catalonia. Catalan Journal of Communication $\&$ Cultural Studies, 8(2), 207-225. https://doi.org/10.1386/cjcs.8.2.207_1

Alsius, S. (1999). Codis ètics del periodisme televisiu (Ethical codes of television journalism). Pòrtic.

Alsius, S., Mauri-Ríos, M., \& Rodríguez-Martínez, R. (2011). Spain: a diverse and asymmetric landscape. In T. Eberwein, S. Fengler, E. Lauk, \& E. Leppik-Bork (Eds.), Mapping media accountability - in Europe and beyond (pp. 155-167). Herbert von Halem Verlag.

Amazeen, M. A. (2020). Journalistic interventions: The structural factors affecting the global emergence of fact-checking.Journalism,21(1), 95-111. https://doi.org/10.1177/1464884917730217

Aznar, H. (1999). Ética y periodismo (Ethics and journalism). Paidós.

Bastian, M. (2019). Media and accountability in Latin America: Framework, conditions, instruments. In Media and Accountability in Latin America (pp. 453-493). Springer VS.

Benson, R. (2018). Can foundations solve the journalism crisis? Journalism, 19(8), 1059-1077. https://doi.org/10.1177/1464884917724612

Bertrand, C. J. (2018). Media ethics and accountability systems. Routledge.

Bruns, A. (2015). Making sense of society through social media. Social media + society, 1(1). https://doi.org/10.1177/2056305115578679

Campos-Domínguez, E. \& Redondo-García, M. (2015). Meta periodismo y transparencia informativa en el periodismo del siglo XXI (Meta-journalism and media transparency in journalism of the 21st century). Obets. Revista de ciencias sociales, 10(1), 185-209. https://doi.org/10.14198/OBETS2015.10.1.07

Carlson, M. \& Lewis, S. C. (2019). Temporal reflexivity in journalism studies: Making sense of change in a more timely fashion. Journalism, 20(5), 642-650. https://doi.org/10.1177/1464884918760675

Cheruiyot, D. (2017). Do bloggers who criticize the press ultimately matter? (Re) defining media accountability in the age of citizen participation. Comunicació, 34(1), 107-121. https://doi.org/10.2436/20.3008.01.157 
Christians, C., Glasser, T. L., McQuail, D., Nordenstreng, K., \& White, R. A. (2009). Normative theories of the media. Journalism in democratic societies. University of Illinois Press.

Codina, L. (2000). Evaluación de recursos digitales en línea: conceptos, indicadores y métodos (Assesment of online digital resources: indicators and methods). Revista Española de Documentación Científica, 23(1), 9-44. https://doi.org/10.3989/redc.2000.v23.i1.315

Craft, S., Vos, T., \& Wolfgang, J. D. (2016). Reader comments as press criticism: Implications for the journalistic field. Journalism, 17(6), 677-693. https://doi.org/10.1177/1464884915579332

Craft, S. \& Vos, T. (2021). The ethics of transparency. In L. Price, K. Sanders, \& W. N. Wyatt (Eds.), The Routledge Companion to Journalism Ethics (pp. 175-183). Routledge.

Culver, K. B. (2017). Disengaged ethics: Code development and journalism's relationship with "the public". Journalism Practice, 11(4), 477-492. https://doi.org/10.1080/17512786.2015.1121788

Diakopoulos, N. \& Koliska, M. (2017). Algorithmic transparency in the news media. Digital Journalism, 5(7), 809-828. https://doi.org/10.1080/21670811.2016.1208053

Díaz-Campo, J. \& Chaparro-Domínguez, M. A. (2020). Periodismo computacional y ética: Análisis de los códigos deontológicos de América Latina (Computational journalism and ethics: An analysis of deontological codes of Latin America). Revista ICONO 14. Revista Científica De Comunicación Y Tecnologías Emergentes, 18(1), 10-32. https://doi.org/10.7195/ri14.v18i1.1488

Díez-Garrido, M., Campos-Domínguez, E., \& Calvo, D. (2019). La transparencia de los partidos políticos como estrategia electoral. Una evaluación de sus promesas y sus páginas web (Political Parties' Transparency As an Electoral Strategy. An Evaluation of Their Promises and Their Websites]). Trípodos, (44), 83-104.

Domingo, D. \& Heikkilä, H. (2012). Media Accountability Practices in Online News Media. In E. Siapera \& A. Veglis (Eds.), The Handbook of Global Online Journalism (pp. 272-289). Wiley-Blackwell.

Eberwein, T., Fengler, S., Lauk, E., \& Leppik-Bork, T. (Eds.) (2011). Mapping media accountability - in Europe and beyond. Helbert Von Halem Verlag.

Eberwein, T., Fengler, S., \& Karmasin, M. (Eds.) (2018). The European Handbook of Media Accountability. Routledge.

Eberwein, T. \& Porlezza, C. (2016). Both sides of the story: communication ethics in mediatized worlds. Journal of communication, 66(2), 328-342. https://doi.org/10.1111/jcom.12216

Fengler, S. (2003). Holding the news media accountable: a study of media reporters and media critics in the United States. Journalism \& Mass Communication Quarterly, 80 (4), 818-832. https://doi.org/10.1177/107769900308000405

Fengler, S., Eberwein, T., Alsius, S., Baisnée, O., Bichler, K., Dobek-Ostrowska, B., ... \& Zambrano, S. V. (2015). How effective is media self-regulation? Results from a comparative survey of European journalists. European Journal of Communication, 30(3), 249-266. https://doi.org/10.1177\%2F0267323114561009

Fengler, S., Eberwein, T., Mazzoleni, G., Porlezza, C., \& Russ-Mohl, S. (2014). Journalists and media accountability. An international study of news people in the digital age. Peter Lang.

Ferrucci, P. (2020). It is in the numbers: How market orientation impacts journalists' use of news metrics. Journalism, 21(2), 244-261. https://doi.org/10.1177/1464884918807056 
Ferrucci, P. (2019). The End of Ombudsmen? 21st-Century Journalism and Reader Representatives. Journalism \& Mass Communication Quarterly, 96(1), 288-307. https://doi.org/10.1177/1077699018805986

Fuente Cobo, C., Martínez Otero J. M., \& del-Prado-Flores, R. (2014). Las audiencias activas en la regulación de los medios: la dialéctica consumidor-ciudadano en España y México (Active audiences in the regulation of the audiovisual media. Consumer versus citizen in Spain and Mexico). Comunicar, 43, 91-99. https://doi.org/10.3916/C43-2014-09

García-Avilés, J. A. (2019). Examining media accountability in online media and the role of active audiences. In T. Eberwein, S. Fengler, \& M. Karmasin (Eds.), Media accountability in the era of post-truth politics: European challenges and perspectives (pp. 270-283). Routledge.

Goodman, L. A. (1961). Snowball Sampling. The Annals of Mathematical Statistics, 32(1), 148-170. https://www.jstor.org/stable/2237615

Hallin, D. C. \& Papathanassopoulos, S. (2002). Political clientelism and the media: southern Europe and Latin America in comparative perspective. Media, Culture \& Society, 24(2), 175-195. https://doi.org/10.1177/016344370202400202

Harcup, T. (2021). Slow journalism as ethical journalism? In L. Price, K. Sanders, \& W. N. Wyatt (Eds.), The Routledge Companion to Journalism Ethics (pp. 77-84). Routledge.

Lee, S. Y. \& Riffe, D. (2017). Who sets the corporate social responsibility agenda in the news media? Unveiling the agenda-building process of corporations and a monitoring group. Public Relations Review, 43(2), 293-305. https://doi.org/10.1016/j.pubrev.2017.02.007

López-Cepeda, A. M., López-Golán, M., \& Rodríguez-Castro, M. (2019). Audiencias participativas en el servicio audiovisual público europeo: Producción de contenidos y derechos de autor (Participatory audiences in the European public service media: Content production and copyright). Comunicar, 60, 93-102. https://doi.org/10.3916/C60-2019-09

Luengo, M., Maciá-Barber, C., \& Requejo-Alemán, J. L. (2017). Evaluating organisational ethics in Spanish news media.Journalism, 18(9), 1142-1162. https://doi.org/10.1177/1464884916643682

Maciá, C. (2006). La figura del defensor del lector, del oyente $y$ del telespectador (The role of the defender of the reader, the listener, and the viewer). Universitas.

Marqués-Pascual, J. \& González-Peláez, M. (2020). Pros y contras del remozado código deontológico de los periodistas catalanes (Pros and cons of the renewed deontological code of Catalan journalists). Estudios sobre el Mensaje Periodístico, 26(1), 207-213. https://doi.org/10.5209/esmp.67300

Masip, P., Ruiz-Caballero, C., \& Suau, J. (2019). Active audiences and social discussion on the digital public sphere. Review article. El profesional de la información, 28(2), e280204. https://doi.org/10.3145/epi.2019.mar.04

Mauri-Ríos, M. \& Ramon-Vegas, X. (2015). Nuevos sistemas de rendición de cuentas de la información periodística. Una exploración del escenario online español (New media accountability systems. Exploration of the Spanish online environment). El Profesional de la Información, 24(4), 380-389. https://doi.org//10.3145/epi.2015.jul.04

Mauri-Ríos, M., Rodríguez-Martínez, R., Figueras-Maz, M., \& Fedele, M. (2018). Press councils as a traditional instrument of media self-regulation: The perceptions of European journalists. Journal ofApplied Journalism \& Media Studies, 7(2), 221-243. https://doi.org/10.1386/ajms.7.2.221_1 
McQuail, D. (1997). Accountability of media to society. Principles and means. European Journal of Communication, 12(4), 511-529. https://doi.org/10.1177/0267323197012004004

Moreno-Gil, V. (2019). Accountability en la prensa española: la publicación de los escritos de rectificación (Accountability in the Spanish press: The publishing of replies). Anàlisi: Quaderns de Comunicació i Cultura, (61), 55-75. https://doi.org/10.5565/rev/analisi.3231

Nelson, J. L. (2019). The next media regime: The pursuit of 'audience engagement' in journalism. Journalism, 22(9), 2350-2367. https://doi.org/10.1177/1464884919862375

Nieborg, D. B. \& Poell, T. (2018). The platformization of cultural production: Theorizing the contingent cultural commodity. New Media \& Society, 20 (11), 4275-4292. https://doi.org/10.1177/1461444818769694

Nolan, D. \& Marjoribanks, T. (2011). Public editors and media governance at The Guardian and The New York Times. Journalism Practice, 5(1), 3-17. https://doi.org/10.1080/17512786.2010.482764

Pastor, L. (2010). Teoría de las cartas al director. La gestión periodística del público (Theory of letters to the director. The journalistic management of the audience). UOC.

Pérez-Díaz, P. L., Zamora Medina, R., \& Arroyas Langa, E. (2020). Between self-regulation and participatory monitoring: comparing digital news media accountability practices in Spain. Media and Communication, 8(2), 112-123. https://doi.org/10.17645/mac.v8i2.2721

Pérez-Soler, S. \& Micó-Sanz, J. (2020). Perfiles profesionales en el periodismo político local en tiempos de redes sociales (Professional profiles in local political journalism in times of social networking websites). Observatorio (OBS*), 14(1), 139-153. Retrieved from http://obs.obercom.pt/index.php/obs/article/view/1523

Plaisance, P. L. (2000). The concept of media accountability reconsidered. Journal of Mass Media Ethics: Exploring Questions of Media Morality, 15(4), 257-268. https://doi.org/10.1207/S15327728JMME1504_5

Raeymaeckers, K. (2005). Letters to the Editor: A Feedback Opportunity Turned into a Marketing Tool. European Journal of Communication, 20(2), 199-221. https://doi.org/10.1177/0267323105052298

Ramon-Vegas, X., Mauri-Ríos, M., \& Alcalá-Anguiano, F. (2016). Transparencia informativa, autorregulación y participación del público (Reporting transparency, self-regulation and readers' participation). Comunicación y Sociedad, (25), 101-125.

Ramon-Vegas, X. \& Mauri-Ríos, M. (2020). Participación de la audiencia en la rendición de cuentas de los medios de comunicación: instrumentos de accountability y su percepción por parte de los ciudadanos españoles (Audience participation for media accountability: instruments and their perception by Spanish citizens). RAEIC, Revista de la Asociación Española de Investigación de la Comunicación, 7(13), 50-76. https://doi.org/10.24137/raeic.7.13.3

Ramon-Vegas, X. \& Rojas-Torrijos, J. L. (2017). Mapping media accountability instruments in sports journalism. El Profesional de la Información, 26(2), 158-171. https://doi.org/10.3145/epi.2017.mar.02

Ramon-Vegas, X., Billings, A. C., \& Rojas-Torrijos, J. L. (2019). Interviews with Former ESPN Ombudsmen / Public Editors Kelly McBride, Robert Lipsyte, and Jim Brady. International Journal of Sport Communication, 12(1), 28-35. https://doi.org/10.1123/ijsc.2018-0127 
Ramon-Vegas, X., Mauri-Ríos, M., \& Díaz-Campo, J. (2020). Instrumentos de rendición de cuentas impulsados por los medios de comunicación: percepción de los periodistas y ciudadanos españoles (In-house media accountability instruments: Spanish journalists' and citizens' perceptions). Revista de Comunicación, 19(1), 221-241. https://doi.org/10.26441/RC19.1-2020-A13

Rodríguez-Martínez, R., López-Meri, A., Merino-Arribas, A., \& Mauri-Ríos, M. (2017). Instrumentos de rendición de cuentas en España. Análisis comparativo en Cataluña, Galicia, Madrid y Valencia (Media accountability instruments in Spain. Comparative analysis in Catalonia, Galicia, Madrid and Valencia). El Profesional de la Información, 26(2), 255-266. https://doi.org/10.3145/epi.2017.mar.12

Rojas-Torrijos, J. L. \& Ramon-Vegas, X. (2017). Accountability in social networks. Ever-evolving stylebooks and feedback through Twitter. Revista Latina de Comunicación Social, (72), 915-941. https://doi.org/10.4185/RLCS-2017-1200-50en

Ruiz Olabuénaga, J. I., Aristegui, I., \& Melgosa, L. (1998). Cómo elaborar un proyecto de investigación social (How to develop a social research Project). Universidad de Deusto.

Serazio, M. (2019). The other "fake" news: Professional ideals and objectivity ambitions in brand journalism. Journalism, 22(6), 1340-1356. https://doi.org/10.1177/1464884919829923

Silva, L. M. \& Paulino, F. (2007). Media Accountability Systems: Models, proposals and outlooks. Brazilian journalism research, 3(1), 137-153 https://doi.org/10.25200/BJR.v3n1.2007.103

Suárez-Villegas, J. C. (2015). Self-regulation of offline and online journalism in Spain in the experience of the Arbitration, Complaints and Ethics Commission. Communication $\mathcal{E}$ Society, 28(3), 135-149. https://doi.org/10.15581/003.28.3.135-149

Usher, N. (2018). Breaking news production processes in US metropolitan newspapers: Immediacy and journalistic authority. Journalism, 19(1), 21-36. https://doi.org/10.1177/1464884916689151

Van Dalen, A. \& Deuze, M. (2006). Readers' advocates or newspapers' ambassadors? European Journal of Communication, 21(4), 457-475. https://doi.org/10.1177/0267323106070011

Wahl-Jorgensen, K. (2002). Understanding the conditions for public discourse: four rules for selecting letters to the editor. Journalism Studies, 3(1), 69-81. https://doi.org/10.1080/14616700120107347

Waisbord, S. (2019). The vulnerabilities of journalism. Journalism, 20(1), 210-213. https://doi.org/10.1177/1464884918809283

Wilkins, L. \& Brennen, B. (2004). Conflicted interests, contested terrain: journalism ethics 000codes then and now. Journalism Studies, 5(3), 297-309.

https://doi.org/10.1080/1461670042000246061 


\section{SOBRE LOS AUTORES}

MARCEL MAURI-RIOS, es periodista, historiador, doctor en Comunicación y profesor en el Departament de Comunicació de la Universitat Pompeu Fabra (UPF) de Barcelona. Miembro del Grup de Recerca en Comunicació Política, Periodisme i Democràcia (POLCOM-GRP) de la UPF. Es profesor de Deontología Periodística en la misma universidad. Sus líneas principales de investigación son la ética periodística y la rendición de cuentas de los medios, el periodismo político y la historia del periodismo. $\mathrm{Ha}$ participado como investigador principal en distintos proyectos competitivos españoles e internacionales relacionados con sus líneas de investigación.

https://orcid.org/0000-0003-2615-8343

XAVIER RAMON-VEGAS, es doctor en Comunicación y profesor del Departamento de Comunicación de la Universitat Pompeu Fabra (UPF). Miembro del Grup de Recerca en Comunicació Política, Periodisme i Democràcia (POLCOM-GRP). Su investigación y docencia se centran en los ámbitos de la ética periodística, la rendición de cuentas de los medios (media accountability) y la comunicación deportiva. Ha participado en tres proyectos competitivos financiados por el Gobierno de España sobre rendición de cuentas de los medios y plataformas de fact-checking.

(iD) https://orcid.org/ 0000-0002-4478-5626

RUTH RODRÍGUEZ-MARTíNEZ, es doctora en periodismo por la Universidad Complutense de Madrid. Forma parte del Grup de Recerca en Comunicació Política, Periodise y Democràcia de investigación POLCOM-GRP de la Universitat Pompeu Fabra (UPF) de Barcelona, donde ejerce como investigadora y profesora. Sus líneas de investigación están vinculadas a la media accountability y la desinformación. Ha coliderado dos proyectos de investigación competitivos financiados por el Gobierno de España (MediaACES y FACCTMedia). Ha participado en el proyecto europeo de MediaAcT y lidera el grupo español del proyecto EurOMo.

https://orcid.org/0000-0001-5633-6126

JESÚS DíAZ-CAMPO, es doctor en Periodismo por la Universidad Complutense de Madrid. Profesor titular en la Universidad Internacional de La Rioja (UNIR), donde desempeña los cargos de Adjunto al Vicerrector de Investigación y secretario del Comité de Ética de la Investigación. Dirige el grupo de investigación Comunicación y Sociedad Digital (COYSODI) y es investigador principal del proyecto Newsharing: Consumo de noticias en medios sociales. Análisis de factores en la selección y difusión de contenidos mediáticos (convocatoria de Proyecto de I+D 2017 Retos).

iD https://orcid.org/0000-0001-5014-8749 